\title{
Limits of coherent supercontinuum generation in normal dispersion fibers
}

\author{
Alexander M. Heidt ${ }^{1}{ }^{*}$, James S. Feehan $^{2}$, and Jonathan H.V. Price ${ }^{2}$ \\ ${ }^{1}$ Institute of Applied Physics, University of Bern, Sidlerstrasse 5, 3012 Bern, Switzerland \\ ${ }^{2}$ Optoelectronics Research Centre, University of Southampton, Southampton SO17 1BJ, United Kingdom \\ *alexander.heidt@iap.unibe.ch \\ Compiled February 5, 2017
}

We study the largely unexplored transition between coherent and noise-seeded incoherent continuum generation in all-normal dispersion (ANDi) fibers and show that highly coherent supercontinua with spectral bandwidths of one octave can be generated with long pump pulses of up to $1.5 \mathrm{ps}$ duration, corresponding to soliton orders of up to $N=600$. In terms of $N$, this corresponds to an approximately 50 times increase of the coherent regime compared to anomalous dispersion pumping. In the transition region between coherent and incoherent spectral broadening we observe the manifestation of nonlinear phenomena that we term incoherent cloud formation and incoherent optical wave breaking, which lead to a gradual or instantaneous coherence collapse of SC spectral components, respectively. The role played by stimulated Raman scattering and parametric four-wave mixing during SC generation in ANDi fibers is shown to be more extensive than previously recognized: their nonlinear coupling contributes to the suppression of incoherent dynamics at short pump pulse durations, while it is responsible for non-phasematched parametric amplification of noise observed in the long pulse regime. We further discuss the dependence of SC coherence on fiber design, and present basic experimental verifications for our findings using single-shot detection of SC spectra generated by picosecond pulses. This work outlines both the further potential as well as the limitations of broadband coherent light source development for applications such as metrology, nonlinear imaging, and ultrafast photonics, amongst others.

(C) 2017 Optical Society of America

OCIS codes: (320.6620) Supercontinuum generation; (190.4370) Nonlinear optics, fibers.

http://dx.doi.org/10.1364/ao.XX.XXXXXX

\section{INTRODUCTION}

Supercontinuum (SC) sources based on nonlinear spectral broadening of ultrashort pulses in specialty optical fibers offer a unique combination of very broad spectral bandwidths, extreme brightness levels, and the possibility of optimizing their properties for specific tasks or pump lasers [1,2]. Thus they have found their way into countless applications, in which the shotto-shot stability of amplitude and phase of the SC pulses, i.e. their degree of temporal coherence, is often a key criterion. Most famously, the generation of SC pulses with a high degree of temporal coherence and octave-spanning spectral bandwidths enabled the realization of stabilized optical frequency combs, which have revolutionized the fields of precision spectroscopy and frequency metrology [3, 4]. In contrast, low temporal coher- ence associated with large pulse-to-pulse fluctuations often plays a precision or resolution limiting role, for example in modalities using the coherence directly as content in the acquired signal, such as optical coherence tomography $[5,6]$ or coherent antiStokes Raman scattering (CARS) spectroscopy [7]. Since spectral amplitude and phase fluctuations also translate into temporal jitter, ultrafast photonics applications usually demand SC sources with a high degree of coherence to enable, for example, beam synchronization and extraction of time-resolved information in multi-beam pump-probe techniques, nonlinear pulse compression, multimodal bio-photonic imaging, or coherent control experiments [8-15].

Consequently, the nonlinear dynamics influencing and limiting temporal coherence have been extensively studied for the 
most common case of SC generation, when femtosecond laser pulses are launched at wavelengths where the group-velocity dispersion (GVD) of the fiber is anomalous. It was found that a competition of coherent and incoherent spectral broadening dynamics, namely soliton fission and modulation instability (MI), governs the coherence properties of the resulting SC $[16,17]$. The dominating set of nonlinear effects is determined by fiber and pump pulse parameters, and in general a pump pulse soliton number of $N \leq 10$ is required to maintain high coherence. In practice, this corresponds to pump pulse durations of $<100 \mathrm{fs}$, while SC generated with longer pulses tend to show strong pulse-to-pulse fluctuations [18-21]. Although fibers with longitudinally varying dispersion were numerically investigated to extend the coherent regime to pump pulses with picosecond durations [22], the necessary careful adjustment of the fiber design to specific pump pulse parameters prevented experimental realization.

In recent years the interest in an alternative pumping approach has increased, where the pulses are launched into fibers with low, flat, and normal GVD over the entire region of interest, i.e. so-called all-normal dispersion (ANDi) fibers. With this fiber design, octave-spanning SC bandwidths can be achieved that were previously only known from their anomalously pumped counterparts. The normal GVD suppresses soliton formation and MI resulting in significant advantages, such as the conservation of ultrashort compressible temporal pulses, uniformly flat spectral profiles, and, most importantly, high stability and coherence for a much larger range of pump pulse parameters [10, 2326]. Even for launched pulses of several hundred femtoseconds duration, ANDi SC can be considered highly coherent, as the well-known nonlinear dynamics in this regime are dominated by self-phase modulation (SPM) and optical wave-breaking (WB) that create new wavelengths coherently with the pump pulse (Fig. 1) [8]. However, so far the fundamental limits of this coherent regime have not been studied. While it is well known that SC generation with long pulse pumping (tens of picoseconds) in the normal GVD regime is dominated by noise-seeded cascaded stimulated Raman scattering (SRS) and is hence incoherent [27], and indications exist that SRS also plays a role in the decoherence mechanism of ANDi fiber SC [28, 29], the transition region between coherent and incoherent spectral broadening in fibers with (all-) normal GVD remains largely unexplored.

Previous studies have shown that the high spectral coherence of ANDi SC sources can be compromised by nonlinear depolarization in weakly birefringent fibers, e.g. through polarization modulation instability [30,31], which severely limits the applicability of ANDi SC sources in the ultrafast photonics applications mentioned above. However, this polarization-related noise can be effectively suppressed by increasing the birefringence of the fiber, e.g. by introducing internal stress in a polarizationmaintaining (PM) fiber design [32]. Hence, it qualifies as an avoidable technical noise source and does not represent a fundamental noise limitation of ANDi SC generation. As PM fiber is the preferred solution for the targeted applications and the experimentally measured SC spectra correspond very well to numerical simulations assuming pulse propagation in a single polarization axis $[30,32]$, this is also the approach we use in this manuscript.

To date, the fundamental noise limitations of ANDi SC generation are not explored and it is unknown, for example, which maximum pump pulse duration can be used to achieve a highly coherent octave-spanning SC, exactly which nonlinear dynamics are at work in the transition region between coherent and

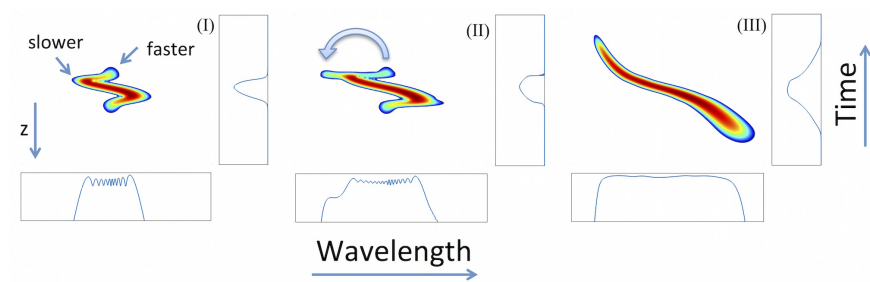

Fig. 1. Schematic overview of coherent SC generation dynamics in normal dispersion fibers using spectrograms with projected axes (bottom: logarithmic spectrum, right: temporal profile) of the pulse at key stages during propagation. $z$ indicates the direction of propagation. (I): SPM broadening. (II) Optical wave breaking on the short wavelength edge: arrow indicates energy transfer into the spectral wing. Wave breaking on the long wavelength edge occurs with further propagation (not shown). (III) Dynamics concluded.

incoherent spectral broadening, and if these dynamics can be influenced by the fiber design. These issues are not only important in the context of SC generation, but are also relevant in other optical systems where a competition between coherent and incoherent dynamics exists, such as in high power ultrafast fiber amplifiers.

Here we address these fundamentally and technologically relevant open questions with comprehensive numerical simulations using a model fiber that closely resembles a commercially available silica ANDi PCF, and present experimental verifications for certain aspects of the numerical findings. The paper is organized as follows. Section 2 introduces the numerical model. In Sec. 3, we investigate the physical processes involved in the decoherence mechanism in ANDi fibers and find that non-phasematched parametric amplification of noise due to a nonlinear coupling of SRS and four-wave mixing (FWM) plays a central role. The coherence limits of SC pulses with spectral bandwidth of one octave are explored in Sec. 4, and we show that high average coherence levels can be maintained with pump pulse durations of up to $\sim 1.5 \mathrm{ps}$, corresponding to initial soliton numbers of up to $N \simeq 600$. We also study the interplay of coherent and incoherent dynamics at high pump peak powers, which results in gradual coherence collapse as the length of the fiber is increased and the manifestation of nonlinear phenomena that we term incoherent cloud formation and incoherent optical wave breaking. In Sec. 5, we examine the mechanisms that suppress incoherent dynamics at high peak powers and short pump pulse durations and that are hence responsible for the excellent coherence properties of ANDI SC sources in this regime. Sec. 6 briefly discusses the impact of pump sources with noise levels exceeding the shot noise limit. Finally, we present experimental observations in Sec. 7 confirming the basic numerical findings, and conclude in Sec. 8.

\section{NUMERICAL MODEL}

Our numerical model is based on the generalized nonlinear Schrödinger equation (GNLSE), which is solved in its frequency domain formulation using a Runge-Kutta in the interaction picture integration method. Additionally we employ an adaptive step size algorithm based on photon number conservation to improve computational speed and ensure sufficient accuracy as the pulse is propagated along the fiber [33]. The implementation of the model is discussed in detail in [34], and a general version of 


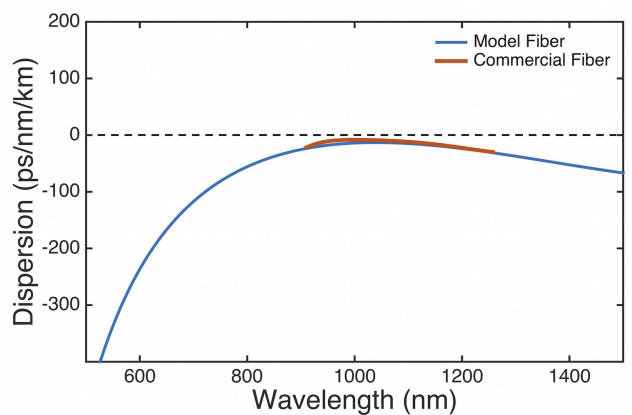

Fig. 2. Dispersion profile of the model fiber used in the simulations with design parameters $\Lambda=1.55 \mu \mathrm{m}$ and $d / \Lambda=0.37$. The measured dispersion of the commercial PCF (NKT NL 1050-NEG) used in the experiments in Sec. 7 is shown for comparison in the range $910-1260 \mathrm{~nm}$.

the simulation code is freely available [35]. The model includes the full dispersion information of the fiber and all relevant nonlinear effects, such as the instantaneous Kerr-type electronic response, delayed Raman contributions, self-steepening and shock-formation terms. Propagation in the forward direction in one polarization axis is assumed. Loss in the fiber is neglected as it does not have a major effect in the wavelength range and for the fiber lengths discussed in this paper.

In order to facilitate the reproduction of our simulations, we assume a simple hexagonal lattice silica PCF with design parameters pitch $\Lambda=1.55 \mu \mathrm{m}$ and relative hole size $d / \Lambda=0.37$, and use an analytical approach to calculate its properties [36] [37]. Fig. 2 shows the corresponding ANDi profile with minimum dispersion wavelength (MDW) at $1040 \mathrm{~nm}$ and peak dispersion parameter $\mathrm{D}=-13 \mathrm{ps} /(\mathrm{nm} \mathrm{km})$. A constant nonlinear parameter $\gamma=18(\mathrm{~W} \mathrm{~km})^{-1}$ is assumed. The fiber design is well suited for the generation of SC spectra with octave-spanning bandwidths [23]. A commercial PCF with similar properties is used in the experiments presented in Sec. 7, and its measured dispersion profile is shown in Fig. 2 as well. We consider pumping with Gaussian input pulses at a central wavelength of $1050 \mathrm{~nm}$ with temporal field envelope $A(T)=\sqrt{P_{0}} \exp \left[-\left(T / T_{0}\right)^{2}\right]$, where $P_{0}$ is the peak power, $T$ is the retarded time in the co-moving frame of reference travelling at the group velocity of the pump wavelength, and $T_{0}$ is related to the full width at half maximum (FWHM) pulse duration $T_{\mathrm{FWHM}}=\sqrt{2 \ln 2} T_{0}$.

The coherence properties of the calculated SC spectra are investigated by including quantum noise terms into the simulations and comparing the results of multiple simulations obtained with different noise seeds. Spontaneous Raman scattering is accounted for directly by a noise term in the GNLSE [17], while input pulse shot noise is modelled semi-classically by adding one photon per mode with random phase on each spectral discretization bin to the input field. The shot-to-shot fluctuations are then characterized using the spectrally resolved modulus of first order coherence at zero path difference

$$
\left|g_{12}(\lambda)\right|=\left|\frac{\left\langle S_{1}^{*}(\lambda) S_{2}(\lambda)\right\rangle}{\sqrt{\left\langle\left|S_{1}(\lambda)\right|^{2}\right\rangle\left\langle\left|S_{2}(\lambda)\right|^{2}\right\rangle}}\right|,
$$

where angle brackets indicate an ensemble average over independently generated SC pairs $\left[S_{1}(\lambda), S_{2}(\lambda)\right]$ obtained from 20 independent simulations. At each wavelength bin, this yields a positive number in the interval $[0,1]$ with the value 1 repre- senting perfect stability in amplitude and phase [18]. Unless otherwise stated we show ensemble averages when displaying numerical results in this paper.

For the interpretation of results we make use of the characteristic length scales and dimensionless parameter [38]

$$
L_{D}=\frac{T_{0}^{2}}{\left|\beta_{2}\right|} ; L_{N L}=\frac{1}{\gamma P_{0}} ; N^{2}=\frac{L_{D}}{L_{N L}},
$$

which are dispersive length $L_{D}$, nonlinear length $L_{\mathrm{NL}}$, and "soliton" number $N$, respectively. $\beta_{2}$ is the second order dispersion coefficient, which assumes the value of $\beta_{2}=7.7 \times 10^{-3} \mathrm{ps}^{2} / \mathrm{m}$ for the pump wavelength of $1050 \mathrm{~nm}$. Although there are no solitons formed in the normal dispersion regime considered here, the usual definition of the parameter $N$ is useful in the interpretation of results.

\section{LOW PEAK POWER PUMPING}

We first consider pumping with relatively low peak power pulses $\left(P_{0}=5 \mathrm{~kW}\right)$ and pump pulse durations in the range $T_{\text {FWHM }}=[50 \mathrm{fs} ; 5 \mathrm{ps}]$. In this regime, the SC spectra are relatively narrow and the different contributing nonlinear effects can be clearly distinguished. Hence this section serves to identify which physical processes are involved in SC generation in ANDi fibers in different regimes of pump pulse durations and how these processes contribute to coherent or incoherent spectral broadening.

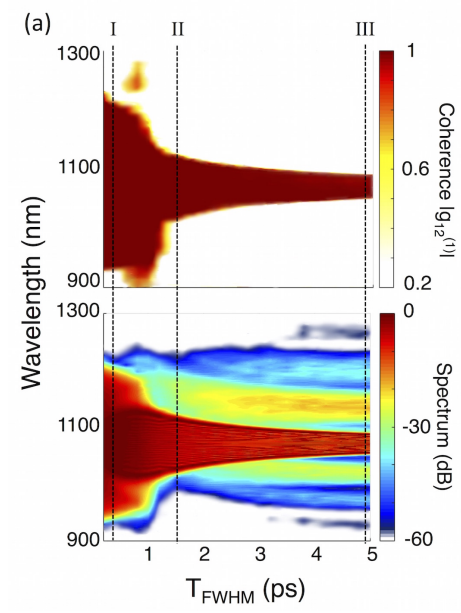

(b)

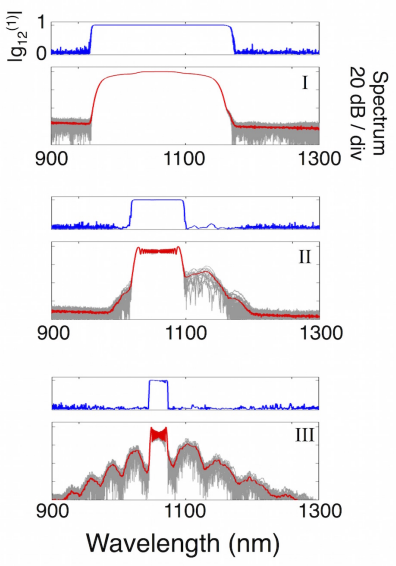

Fig. 3. a) Supercontinuum mean spectral intensity (bottom) and corresponding spectral coherence (top) generated by a 5 $\mathrm{kW}$ peak power pump pulse after $1 \mathrm{~m}$ propagation as a function of input pulse duration $T_{\text {FWHM }}$ b) Detail from a) marked in positions I, II, and III corresponding to $200 \mathrm{fs}, 1500 \mathrm{fs}$ and $5000 \mathrm{fs}$ pulse durations, respectively. The mean spectrum is displayed in red, while the intensity fluctuations are visualized by displaying an ensemble of 20 independent simulations with different noise seeds in grey.

Figure 3 shows the logarithmic spectra and the corresponding coherence as a function of $T_{\mathrm{FWHM}}$ for a fiber length of $1 \mathrm{~m}$ using colour map density plots as well as in more detail for three representative cases. For sub-picosecond pump pulse durations, the well-understood coherent spectral broadening dynamics dominated by SPM and WB already described in Fig. 1 are at work, resulting in uniform temporal and spectral profiles as well as perfect coherence of the final SC spectrum. 
For $T_{\mathrm{FWHM}} \approx 1$ ps the spectra become abruptly narrower as the spectral side lobes generated by WB disappear. This is due to the fact that the characteristic length scale $L_{\mathrm{WB}}$ on which WB occurs becomes larger than the considered fiber length of $1 \mathrm{~m}$. An analytical expression for $L_{W B}$ has been derived [39]

$$
L_{\mathrm{WB}} \approx 1.1 \frac{L_{D}}{N}=1.1 T_{0} \sqrt{\frac{1}{\gamma P_{0} \beta_{2}}},
$$

which becomes larger than $1 \mathrm{~m}$ for $T_{\text {FWHM }}>1.1 \mathrm{ps}$ and hence agrees well with the numerical result. Without the smoothing action of $\mathrm{WB}$, the central spectrum starts showing the oscillatory structure associated with purely SPM-induced broadening, and a relatively broad incoherent peak appears at the long-wavelength side, which is clearly visible in the detail for $T_{\mathrm{FWHM}}=1500 \mathrm{fs}$. It is red-shifted from the central spectrum by an angular frequency of $\Omega_{R}=\omega_{p}-\omega_{s}=2 \pi \times 13.2 \mathrm{THz}$, and can hence be attributed to stimulated Raman scattering (SRS). Here $\omega_{\alpha}(\alpha=p, s)$ are the carrier-wave angular frequencies of pump and Stokes wave, respectively. For even longer pump pulse durations, cascaded Stokes and anti-Stokes peaks appear and progressively dominate the spectrum. Only the spectral range created by SPM-induced broadening exhibits high coherence, while both Stokes and anti-Stokes peaks show large fluctuations in amplitude and phase from shot to shot.
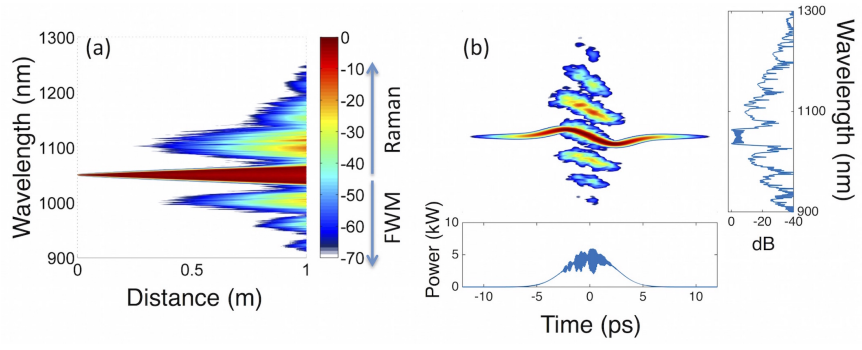

Fig. 4. a) Single-shot spectral evolution of a $5 \mathrm{~kW}, 5000 \mathrm{fs}$ pump pulse over $1 \mathrm{~m}$ propagation distance. $\mathrm{b}$ ) Spectrogram of the pulse at the exit of the fiber, with projected axes showing logarithmic spectrum (right) and pulse profile (bottom).

Raman anti-Stokes components are rarely observed in fibers, but play a crucial role in the decoherence mechanism of normal dispersion SC, because they provide an effective noise amplification mechanism at wavelengths shorter than the pump. We therefore investigate their origin further in Fig. 4, which illustrates the spectral evolution of the 5 ps pump pulse over $1 \mathrm{~m}$ propagation distance as well as the spectrogram at the exit of the fiber. The appearance of every order of cascaded Raman Stokes peak is almost immediately followed by the creation of the corresponding anti-Stokes wave. The growth of the anti-Stokes peaks at $\omega_{a, i}=\omega_{p}+i \times \Omega_{R}$ can be attributed to Raman assisted parametric four-wave mixing (FWM) with the pump pulse. Although the all-normal dispersion profile of the fiber excludes phase-matching of Stokes and anti-Stokes components with the pump wave, the combined action of SRS and FWM can provide efficient parametric amplification of the anti-Stokes component even when the linear phase mismatch is large. This effect was first predicted by Bloembergen and Shen for free space beams $[40,41]$, and experimentally demonstrated in optical fibers by Coen et al. [27, 42]. Also in our simulation we confirmed that the Raman gain is essential for the creation of the anti-Stokes and higher order Stokes peaks: if the Raman gain term is switched off at any position along the fiber, spectral broadening stops immediately. As expected, parametric FWM by itself is therefore not efficient due to phase mismatch. The ratio of Stokes and anti-Stokes powers under non-phase-matched conditions can be estimated to be [42-44]

$$
\frac{P_{a}}{P_{s}} \simeq\left|\frac{\gamma q P_{0}}{2 \gamma q P_{0}+\beta_{2} \Omega_{R}^{2}}\right|^{2},
$$

where $q=\left(1-f_{R}\right)+f_{R} \tilde{\chi}_{R}^{(3)}\left(-\Omega_{R}\right)$ contains the complex Raman susceptibility $\tilde{\chi}_{R}^{(3)}(\Omega)$ and the fractional contribution of the Raman effect to the total nonlinear response of silica glass, $f_{R} \simeq$ 0.18. Applied to our conditions and with $\tilde{\chi}_{R}^{(3)}\left(-\Omega_{R}\right)=-1.38 i$, Eq. 4 yields an expected ratio of $P_{a} / P_{S} \simeq 0.14$, which indicates strong coupling and efficient energy transfer from the pump to shorter wavelengths. This is in good agreement with our numerical results for pulse durations which develop clearly distinguishable Stokes and anti-Stokes components, i.e. $T_{\mathrm{FWHM}}>3$ ps.

The Stokes and anti-Stokes components develop as separate side pulses near the temporal center of the pump pulse where its instantaneous power is largest, as shown in the spectrogram in Fig. 4 (b). In this region the pump exhibits a linear chirp due to SPM, which is transferred to the Stokes and anti-Stokes pulses due to cross phase modulation (XPM). The temporal overlap of the different wavelength components leads to the noise-like interference structures visible on the temporal pulse profile.

Fig. 4 (b) also illustrates that temporal walk-off is negligible due to the low and flat dispersion profile of the ANDi fiber considered here. The walk-off length $L_{W}=T_{0} /|d|$, where $d=$ $v_{g, p}^{-1}-v_{g, s}^{-1} \simeq 0.7 \mathrm{ps} / \mathrm{m}$ is related to the group velocities $v_{g}$ of pump and Stokes pulse, becomes as large as $6 \mathrm{~m}$ for $T_{\mathrm{FWHM}}=5$ ps. Even for sub-picosecond pulses, the spectral broadening due to SPM/WB leads to fast dispersive temporal broadening such that the effective $L_{W}$ is much longer than the considered fiber length of $1 \mathrm{~m}$. The long $L_{W}$ is conducive for the energy exchange between the overlapping wavelength components, and hence continued development of cascaded Stokes and antiStokes pulses is expected if the fiber length is increased.

Clearly, the broadening dynamics are governed by a competition between SPM/WB on one side and SRS/FWM on the other side. The coherence properties of the generated spectra critically depend on the dominating set of nonlinear effects. While SPM and WB are seeded by wavelength components within the pump pulse itself and therefore maintain its coherence properties, SRS can also build up from noise if its gain bandwidth remains unseeded. This amplified noise is random and hence incoherent with the pump, and in conjunction with Raman assisted FWM can quickly spread throughout the SC spectrum. It is important to note that the observed generation of strong anti-Stokes components is crucial for the understanding of the decoherence mechanism in normal dispersion SC, since it is the dominating effect for noise amplification at wavelengths shorter than the pump. The interplay of SRS with FWM can therefore be identified as the main mechanism of decoherence in normal dispersion fibers.

\section{HIGH PEAK POWER PUMPING}

Having identified the main physical effects involved in the decoherence mechanism, we now increase the peak power of the pump pulses to $P_{0}=100 \mathrm{~kW}$ in order to investigate how these processes manifest themselves in the generation of supercontinuum pulses with spectral bandwidths approaching one octave. 


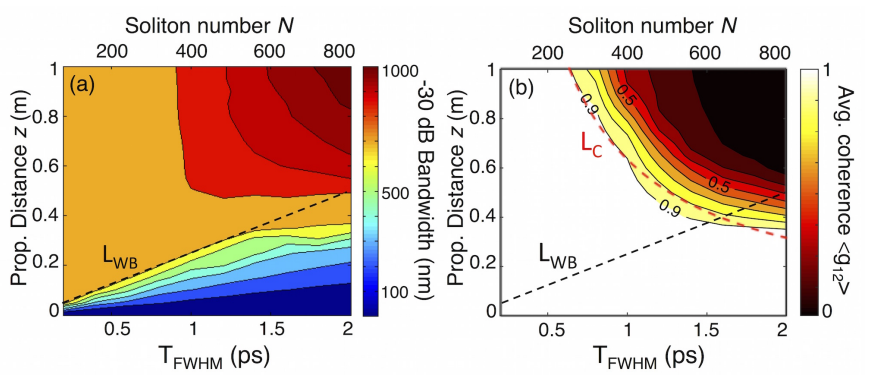

Fig. 5. (a) - $30 \mathrm{~dB}$ spectral bandwidth and (b) average spectral coherence of SC pulses generated with $100 \mathrm{~kW}$ peak power pump pulses as a function of pump pulse duration and propagation distance. The corresponding soliton numbers are given on the top $x$-axis. The analytical expressions for the wave breaking distance $L_{\mathrm{WB}}$ (Eq. 3) and the coherence length $L_{c}$ (Eq. 11) are indicated as dashed lines.

Figure 5 provides an overview of the evolution of spectral bandwidth (a) and average spectral coherence (b) in the range $T_{\mathrm{FWHM}}=[50 ; 2000] \mathrm{fs}$ and propagation distance $z=[0 ; 1] \mathrm{m}$. This corresponds to initial soliton numbers of approximately $N=[20 ; 800]$. Here the spectrally averaged coherence is defined as $\left\langle g_{12}\right\rangle=\int\left|g_{12}(\lambda)\right||S(\lambda)|^{2} d \lambda / \int|S(\lambda)|^{2} d \lambda$. The analytical wave breaking distance $L_{W B}$ obtained with Eq.(3) is also indicated. From this figure we can distinguish three regimes for the SC generation process:

- $T_{\text {FWHM }}<1$ ps $(N \leq 400)$ : this is the most widely considered regime to date and is characterized by the generation of highly coherent spectra over a large parameter space. The SC are generated by the well-known dynamics dominated by SPM/WB, and it is clear that $L_{\mathrm{WB}}$ plays the role of a characteristic length scale after which the coherent SC generation process is concluded and the spectral bandwidth remains constant. The maximum spectral bandwidth in this coherent regime $\Delta \omega_{\text {coh }}$ is independent of $T_{\text {FWHM }}$, and its dependence on other fiber and pulse parameters can be shown to be [8]

$$
\Delta \omega_{\text {coh }} \propto \sqrt{\frac{\gamma P_{0}}{\beta_{2}}} .
$$

In a given fiber, $\Delta \omega_{\text {coh }}$ is therefore solely dependent on $P_{0}$. However, the required fiber length to achieve this maximum bandwidth increases with pump pulse duration since $L_{\mathrm{WB}} \propto T_{0}$. The quantity

$$
g_{\mathrm{WB}}=1 / L_{\mathrm{WB}}
$$

can therefore be regarded as an effective "gain" of the coherent SC generation process indicating the strength or speed with which it occurs. The coherence length $L_{c}$, which we define as the maximum propagation distance for which $\left\langle g_{12}\right\rangle>0.9$, i.e.

$$
L_{c}=\max \left(\left.z\right|_{\left\langle g_{12}\right\rangle>0.9}\right),
$$

is generally very large in this regime. Only for extended propagation distances $z \gg L_{\mathrm{WB}}$ can the onset of coherence degradation be observed. Note that the simulated value of $L_{c}$ is equal to the contour line for $\left\langle g_{12}\right\rangle=0.9$ indicated in Fig. 5 (b). The dashed red line in the same figure stems from the analytic expression Eq. (11) discussed in Sec. 5.
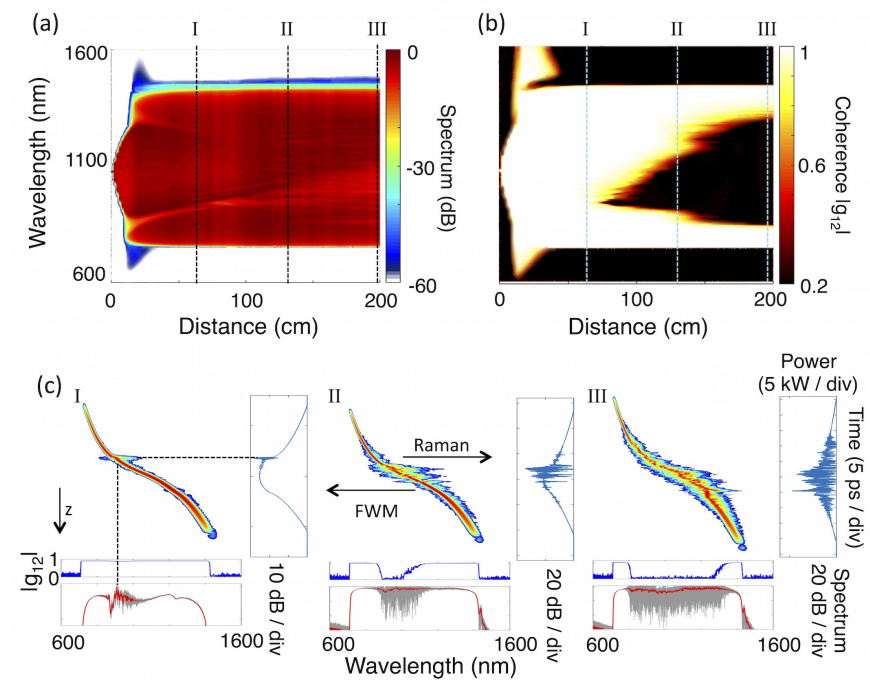

Fig. 6. Evolution of (a) mean spectral intensity and (b) spectral coherence of a $100 \mathrm{~kW}, 600 \mathrm{fs}(N \simeq 250)$ pump pulse over 2 $\mathrm{m}$ of propagation distance. (c) Single-shot spectrogram with projected axes showing spectral coherence, mean spectrum (red), spectral fluctuations (grey) as well as the single-shot temporal pulse profile at propagation distances marked I, II, and III in (a) and (b) corresponding to $55 \mathrm{~cm}, 130 \mathrm{~cm}$, and 200 $\mathrm{cm}$, respectively. $z$ indicates the direction of propagation.

- 1 ps $<T_{\text {FWHM }}<1.5$ ps $(400<N<600): L_{c}$ decreases rapidly and becomes comparable to $L_{W B}$. Hence the parameter space in which full spectral bandwidth and high coherence are obtainable simultaneously becomes very narrow and careful adjustment of the fiber length is required to maintain both. Nevertheless, octave spanning SC with good coherence properties should be practically achievable with pump pulse durations of up to 1.5 ps $(N \simeq 600)$. In terms of $N$, this corresponds to an approximately 50 times increase of the coherent regime compared to anomalous dispersion pumping [17].

- $T_{\mathrm{FWHM}}>1.5$ ps $(N>600): L_{c}$ becomes smaller than $L_{\mathrm{WB}}$, i.e. degradation of coherence already occurs before the coherent SC generation dynamics are concluded. Therefore, coherent SC can only be realized by accepting significantly narrower spectral bandwidths than in the other regimes discussed above. The bandwidth can be enhanced by choosing longer fiber lengths, but this is paid for by a significant degradation of the average coherence.

The discussion above indicates that there might be differences in the interplay between coherent and incoherent spectral broadening dynamics depending on whether coherence degradation begins before or after the coherent WB dynamics are fully concluded. We could indeed identify two different decoherence mechanisms, which we term incoherent cloud formation and incoherent optical wave breaking. The dominating mechanism depends on the relation of $L_{W B}$ and $L_{c}$. In the following sections, we therefore analyze the spectro-temporal evolution of a $600 \mathrm{fs}$ and a 1.6 ps pump pulse along the fiber.

\section{A. $\mathrm{L}_{\mathrm{WB}}<\mathrm{L}_{\mathrm{c}}$ : Incoherent cloud formation}

Figure 6 shows the evolution of mean spectral intensity and coherence of a $100 \mathrm{~kW}, 600 \mathrm{fs}$ pulse $(N \simeq 250)$ over $2 \mathrm{~m}$ of fiber, as 
well as detailed projected axes spectrograms of the pulse at three representative positions. The spectrograms and the projected temporal profile are calculated from a single-shot simulation, while the spectral projection uses the full information of the multi-shot ensemble used to calculate $\left|g_{12}(\lambda)\right|$, i.e. it displays the mean spectrum, spectral shot-to-shot fluctuations, and the corresponding spectrally resolved coherence function. This allows us to correlate the coherence degradation, which is an ensemble phenomenon, to the occurrence of specific spectrotemporal features within a single pulse.

As mentioned above, for the parameters used in Fig. 6 coherent SC generation dynamics are fully developed before any coherence degradation becomes significant. The spectrum assumes its maximum bandwidth after $L_{\mathrm{WB}} \simeq 13 \mathrm{~cm}$, spanning over one octave from 710 to $1420 \mathrm{~nm}$, and remains unchanged with further propagation. The spectrogram has the characteristic " $\mathrm{S}$ "-shape, in which each wavelength component is located at a unique temporal position within the pulse. Hence the pulse is initially free of interference fine structures.

Only after $z>4 L_{\mathrm{WB}}$ (position (I)) does the first signature of noise-seeded SRS appear. Note that this first SRS signature is not simply located at the maximum temporal peak intensity, but at the maximum of the spectro-temporal intensity distribution, marked in spectrogram (I). This corresponds to the position of the outermost blue-shifted SPM peaks in this case. Upon further propagation, each section of the main pulse creates Raman components on its long-wavelength side independently. The emergence of these SRS components continues to follow the spectro-temporal intensity profile of the pulse, i.e. they spread towards the peak and leading edge of the pulse (position (II)). Analogous to the low peak power case, Raman assisted FWM quickly spreads the noise also to the short-wavelength side. The presence of SRS and FWM components and their overlap with the main pulse now cause noisy interference structures and large pulse-to-pulse fluctuations in both spectral and temporal domain. Spectrally these dynamics correspond to a gradual coherence collapse, starting from the outermost blue-shifted SPM peaks spreading towards the spectral edges, predominantly towards longer wavelengths. In contrast to the low power case, the SRS/FWM components cannot be considered as distinct side pulses, but rather merge with the main pulse until an incoherent and noisy "cloud" forms around its formerly coherent core (position (III)). As an important conclusion from this study we note that even a fully developed and highly coherent SC spectrum can experience a coherence collapse in parts or all of its spectral bandwidth if it is propagated over extensive lengths of fiber.

\section{B. $\mathbf{L}_{W B}>\mathbf{L}_{\mathbf{c}}$ : Incoherent optical wave breaking}

Figure 7 is equivalent to Fig. 6, but considers a longer pump pulse duration of $1600 \mathrm{fs}(N \simeq 650)$. Decoherence effects therefore become significant before the coherent SC generation dynamics are fully concluded. Spectrogram (I) shows the pulse at $z \simeq 0.75 L_{\mathrm{WB}}$. Since the dispersion profile of the fiber is steeper for short wavelengths than for long wavelengths (compare Fig. 2), coherent WB is already in progress on the trailing pulse edge, but has not yet begun on the leading edge. Instead, a SRS pulse develops at its characteristic frequency $\omega_{R}=w_{\mathrm{SPM}}-\Omega_{R}$ at the outermost red-shifted SPM peak, which coincides with the highest spectro-temporal intensity section of the pulse. The corresponding FWM peak can also readily be identified.

Due to the normal GVD of the fiber, the red-shifted Raman pulse propagates faster than the SPM components at $1200 \mathrm{~nm}$,
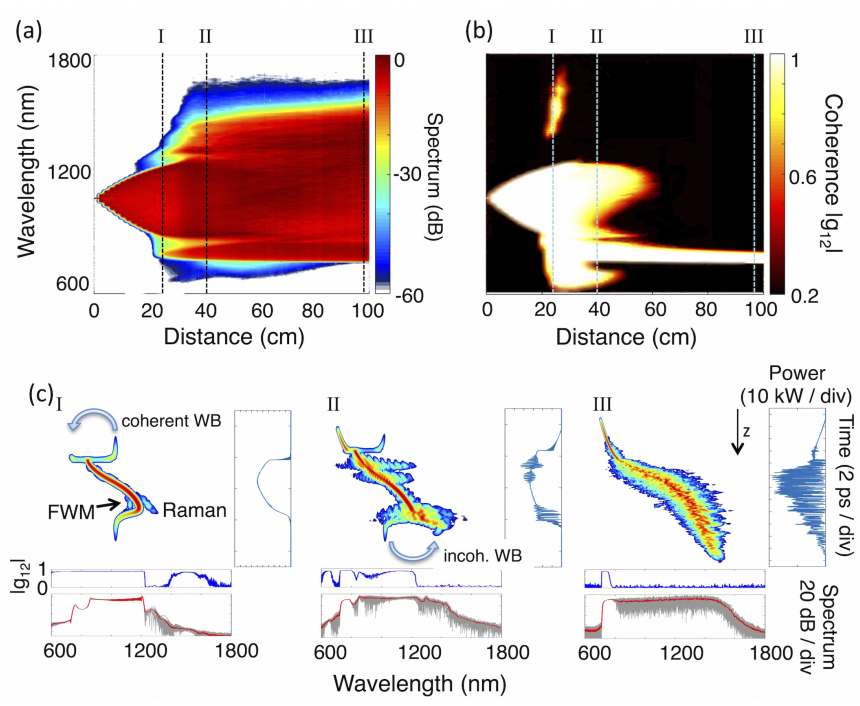

Fig. 7. Evolution of a $100 \mathrm{~kW}, 1600 \mathrm{fs}$ pump pulse $(N \simeq 650)$ over $1 \mathrm{~m}$ propagation distance in a representation equivalent to Fig. 6. Positions I, II, and III correspond to $25 \mathrm{~cm}, 40 \mathrm{~cm}$, and $100 \mathrm{~cm}$ propagation distance, respectively.

which in turn propagate faster than the leading pulse edge at $1050 \mathrm{~nm}$. Eventually, this leads to a temporal overlap of all three components and the occurrence of what is best described as incoherent optical wave-breaking, characterized by nonlinear mixing between the SPM components, SRS pulse, and leading pulse edge. This situation is illustrated in spectrogram (II) at $z \simeq 1.2 L_{\mathrm{WB}}$. Similar to coherent $\mathrm{WB}$, an energy transfer process into the spectral wing takes place (indicated by the arrow), but as a result of the involvement of the noise-seeded SRS pulse in this process the long wavelength edge is generated incoherently. Simultaneously, the SRS / FWM decoherence mechanism already discussed in Fig. 6 is active as well, evident by gradual noiseseeded frequency generation near the outermost blue-shifted SPM peak and at the temporal peak intensity. Hence, coherence is never established on the long wavelength side of the spectrum. In the part of the pulse unaffected by incoherent wave breaking, coherence collapses gradually with further propagation, starting from wavelengths associated with the highest spectro-temporal intensities, in this case both the outermost red- and blue-shifted SPM peaks. Note that here we observe two different forms of decoherence mechanism at the same time: instantaneous decoherence during incoherent $\mathrm{WB}$, where wavelength components are directly generated incoherently, and gradual decoherence in the remaining spectrum due to the continuous amplification of shot noise, which results in progressive coherence collapse over an extended propagation distance.

At the exit of the fiber (spectrogram (III)), the mean spectrum is almost identical to the spectrum generated with the much shorter $600 \mathrm{fs}$ pump pulse in Fig. 6, except for the development of a low-level tail on the long wavelength edge caused by incoherent WB. In fact, this tail is responsible for the observed increase in spectral bandwidth in the incoherent region of Fig. 5 (a). The familiar incoherent "cloud" has formed around the pulse and all but the shortest wavelengths have lost their coherence. The short wavelengths are contained in a long temporal tail and propagate at the back of the pulse with the slowest velocity. Their spectro-temporal intensity is low and they are unaffected by SRS/FWM components in other parts of the pulse. 
Hence, in all investigated cases the short wavelength coherence is maintained over large propagation distances.

We have demonstrated in this section that the well-known coherent SC generation dynamics in normal dispersion fibers, which are governed by the interplay of SPM and WB, are substantially modified by the occurrence of SRS and Raman-assisted FWM if long fiber lengths or long pump pulse durations are considered, leading to coherence collapse in parts or in the entire pulse. However, in all investigated cases of high peak power pumping the resulting mean spectrum at the fiber exit, which would be measured by a slow spectrometer averaging over many shots, is practically undistinguishable from a perfectly coherent spectrum generated with shorter fibers or pump pulses. The characteristic features of broadband normal dispersion SC, such as flat top, smooth profile, and steep edges, are conserved during coherence collapse. In an experiment it is therefore impossible to deduct any meaningful conclusion about the coherence properties of the generated continuum by measuring the spectrum at the fiber output alone. However, the coherence can be evaluated using single-shot spectral measurements and statistically analyzing spectral fluctuations, as shown in Sec. 7. In contrast, in the low power case coherent and incoherent parts of the pulse have clearly distinguishable spectral features.

\section{SUPPRESSION OF INCOHERENT DYNAMICS AT SHORT PUMP PULSE DURATIONS}

The numerical simulations clearly show a dominance of the coherent dynamics at short pulse durations and a complete absence of any noise amplifying effects for fairly long propagation distances. This is a somewhat puzzling observation since one could expect also a significant gain of SRS at the high pump peak powers employed for SC generation. Let us consider the standard gain coefficient for the Stokes wave at the peak of the Raman amplification spectrum at $\Omega=-\Omega_{R}$ given by [38]

$$
g_{s}=-2 \gamma f_{R} \operatorname{Im}\left(\chi_{R}^{(3)}\left(-\Omega_{R}\right)\right) \simeq 0.5 \gamma .
$$

For our conditions, the peak Raman gain $g_{R}=g_{s} P_{0}$ assumes an enormous value of $g_{R}=900 / \mathrm{m}$ for $P_{0}=100 \mathrm{~kW}$, and is still respectable at $P_{0}=5 \mathrm{~kW}$ with $g_{R}=45 / \mathrm{m}$. Using the characteristic length scale $L_{R}=1 / g_{R}$, one would expect SRS signatures to appear within the first few centimeters of propagation. However, in the simulations SRS needs significantly longer to build up; short pump pulses with $T_{\mathrm{FWHM}} \leq 1$ ps could be propagated over distances of $1 \mathrm{~m}$ and more without any SRS development. This suggests that an extremely effective mechanism must be at work to suppress the Raman gain, especially at short pulse durations. Note that $L_{\mathrm{R}}<L_{W}$, so walk-off between pump and Raman pulse does not play a significant role. Also dispersive pulse broadening during the SC generation process cannot explain the Raman gain suppression, since $P_{0}$ only drops by a factor of 2 at $z=L_{\mathrm{WB}}[39]$.

After a more detailed consideration, we find that the nonlinear coupling of SRS and parametric FWM already identified in Sec. 3 to be responsible for the development of anti-Stokes Raman components also contributes significantly to the suppression of the Raman gain. The interaction between these two nonlinear processes leads to an explicit dependence of the Raman gain on the ratio of chromatic dispersion to nonlinearity, expressed in the mixed parametric-Raman peak gain coefficient [42-44]

$$
g_{s}^{*}=2 \gamma \operatorname{Re}(\sqrt{K(2 q-K)}),
$$
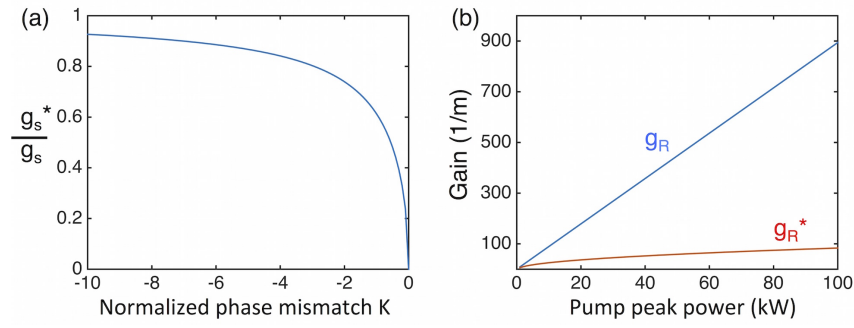

Fig. 8. (a) Normalized Raman peak gain coefficient $g_{s}^{*} / g_{s}$ in dependence of the parameter $\mathrm{K}$ in the normal dispersion regime. (b) Gain comparison of noise-amplifying nonlinear effects for the investigated fiber parameters in dependence of the pump pulse peak power. Displayed are standard Raman gain $g_{R}=g_{s} P_{0}$ and mixed parametric-Raman gain $g_{R}^{*}=g_{s}^{*} P_{0}$.

where $K=-\beta_{2} \Omega_{R}^{2} /\left(2 \gamma P_{0}\right)$ is the linear phase mismatch between pump and (anti-) Stokes wave normalized to the nonlinear contribution to the mismatch. Fig. 8 (a) shows $g_{s}^{*}$ as a function of $K$ in the normal dispersion regime $(K<0)$, normalized by the standard peak Raman gain coefficient $g_{s}$ from Eq. (8). $g_{s}^{*}$ converges towards $g_{s}$ when FWM is negligible, i.e. when the parametric process is completely phase mismatched $(K \rightarrow-\infty)$. For $|K|<1$, which corresponds to the case of low dispersion and high peak powers usually employed for SC generation, the Raman gain is significantly suppressed. This is illustrated more concretely in Fig 8 (b), where the standard Raman gain $g_{R}=g_{s} P_{0}$ and the mixed parametric-Raman gain $g_{R}^{*}=g_{s}^{*} P_{0}$ are directly compared for the fiber parameters and pump powers investigated in the simulations. It is evident that the coupling of SRS and FWM causes a gain saturation effect such that the gain for these noise-amplifying nonlinear dynamics is drastically reduced at high pump powers, for example $g_{R}^{*}<1 / 10 g_{R}$ at $P_{0}=100 \mathrm{~kW}$.

This insight can be related to the numerical simulations of the previous sections by examining its impact on the coherence length $L_{c}$. The simulations have shown that SC generation in normal dispersion fibers is governed by the competition of coherent (SPM/WB) and incoherent (SRS/FWM) broadening dynamics. Hence it is a reasonable assumption that $L_{c}$ depends on their relative importance, expressed by the ratio of their characteristic length scales

$$
L_{c} \propto \frac{L_{R}^{*}}{L_{\mathrm{WB}}},
$$

where $L_{R}^{*}=1 /\left(g_{s}^{*} P_{0}\right)$ and $L_{\mathrm{WB}}$ is defined in Eq. (3). As $f_{r}$ is small, we can expand Eq. (9) in a binomial expansion in the limit of $|K| \ll 1$ and obtain the simple expression $1 / L_{R}^{*} \simeq$ $1.38 f_{r} \Omega_{R} \sqrt{\gamma P_{0} \beta_{2}}$, such that

$$
L_{c}=\xi \frac{1}{f_{r} \Omega_{R} T_{0}},
$$

where $\xi$ has the unit of a length scale and is in general a function of pump power and fiber parameters, i.e. $\xi=\xi\left(\beta_{2}, \gamma, P_{0}\right)$. Hence, for a given fiber design and peak power our analytical approach predicts an inversely proportional relation between coherence length and input pulse duration. This relation for $L_{c}$ can now be compared to the results of the simulations in Fig. 5 (b), where the average coherence $\left\langle g_{12}\right\rangle$ for $P_{0}=100 \mathrm{~kW}$ is shown in a contour plot as a function of $z$ and $T_{\text {FWHM }}$. Eq. (11) with a fitted value of $\xi=8 \mathrm{~m}$ is plotted as a red dashed line, and should 
be compared to the simulated contour line for $\left\langle g_{12}\right\rangle=0.9$, according to our definition of $L_{c}$ in Eq. (7). Clearly, our analytic expression for the coherence length in Eq. (11) reproduces very well the trends observed in the simulation, confirming the validity of our approach.

Two important conclusions can be drawn from this brief analysis : (i) a flat and low normal dispersion is conducive for a significant reduction of the gain for noise-amplifying nonlinear effects, which saturate at high peak powers and nonlinearity, as demonstrated with Eq. (9) and Fig. 8. Interestingly, a comparison with Eq. (5) reveals that the same conditions are required for maximizing the spectral bandwidth of the coherent SC. Hence an ANDi fiber design with low and flat normal dispersion profile not only maximizes the achievable SC bandwidth, but also the suppression of noise-seeded dynamics and hence temporal coherence. (ii) Incoherent dynamics are further suppressed at short pump pulse duration due to a competition with the coherent dynamics, as expressed in Eqs. (10) and (11). Physically, the competition between these two sets of nonlinear effects can be understood by revisiting the significant spectro-temporal reshaping of the pulse during the initial phase of propagation. As seen in Sec. 4, the Raman gain is largest near the sections of the pulse with highest spectro-temporal intensity. In the initial phase of propagation, this corresponds to the positions of the outermost spectral maxima created by SPM. For short pump pulses and hence small $L_{W B}, S P M$ broadening is fast and the spectral maxima shift rapidly in spectral and relative temporal position, such that the position of the maximum Raman gain shifts rapidly as well within the spectrogram. Raman amplified noise therefore does not accumulate at one single point in the spectrogram, but instead is spread out over a wide bandwidth and does not become significant. Only when SPM broadening and the associated spectro-temporal reshaping is slowed down, either by large $L_{\mathrm{WB}}$ or at large propagation distances $z>L_{\mathrm{WB}}$, Raman amplified noise has a chance to build up exponentially.

The combined action of both mechanisms explains the excellent coherence properties of supercontinua generated in ANDi fibers with high power sub-picosecond pump pulses and moderate fiber lengths. Note, however, that the validity of Eq. (11) is limited by the approximation $|K| \ll 1$ to low dispersion regions and very high pump powers. It would be useful to develop generally valid expressions for estimating the coherence of the generated SC from fiber and pump pulse parameters alone, but this would require a more detailed analysis of the dynamics involved, which is outside the scope of this paper and will be pursued elsewhere. Nevertheless, it is clear that the relation between $L_{\mathrm{WB}}$ and $L_{R}^{*}$ is of central importance and can be used together with the identified decoherence mechanisms to interpret experimental results, which will be shown in the following section.

\section{PUMP SOURCES WITH NOISE LEVELS EXCEEDING THE SHOT NOISE LIMIT}

The primary purpose of this paper is to explore the fundamental noise limitations of ANDi SC sources, and hence we assume shot noise limited pump lasers in the simulations presented above. This is a valid approximation as the range of pump pulse durations considered by us implies the use of mode-locked lasers, which can exhibit excellent low-noise performance [45]. Nevertheless, since our results indicate that the coherence loss is initiated by amplification of shot noise via SRS followed by Raman-assisted FWM, it is possible to extend our findings to

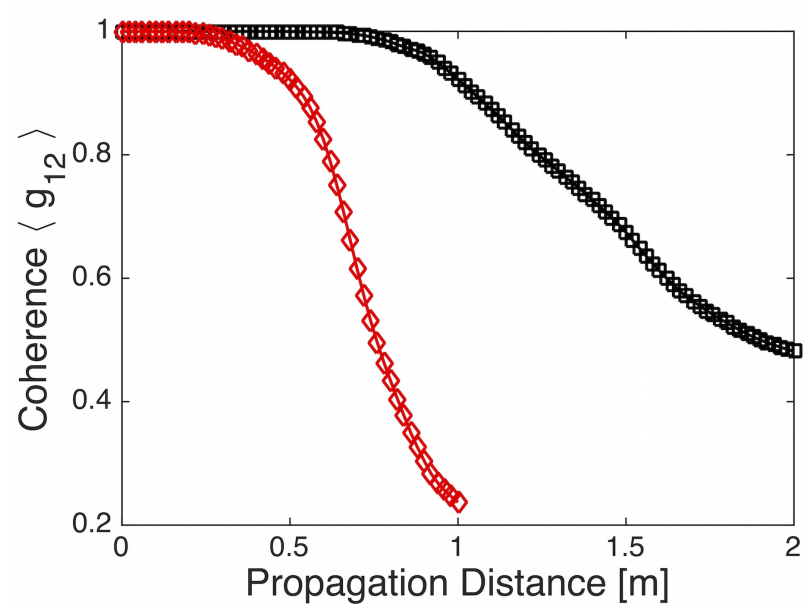

Fig. 9. Evolution of average coherence with propagation distance through the fiber for a $100 \mathrm{~kW}, 600 \mathrm{fs}$ pulse assuming shot noise limited input (black squares) and an additional broadband excess noise component of $25 \mathrm{~dB}$ above shot noise limit (red diamonds).

pump sources with noise levels exceeding the shot noise limit. The growth of the Raman Stokes component is governed by [38]

$$
\frac{d I_{S}}{d z} \propto I_{p} I_{S}
$$

where $I_{p, s}$ are pump and Stokes intensity, respectively. Therefore, any additional seed noise within the Raman amplification bandwidth (e.g. due to amplified spontaneous emission from the pump laser system) would lead to a faster growth of the Stokes component and, consequently, also to a faster loss of coherence than presented in our simulations. This is illustrated in Fig. 9, where the evolution of the average coherence $\left\langle g_{12}\right\rangle$ with propagation distance is shown for two sets of simulations of a $100 \mathrm{~kW}$, $600 \mathrm{fs}$ pump pulse. One set assumes shot noise limited input, the second set assumes an additional broadband input noise component of $25 \mathrm{~dB}$ above shot noise limit. Incoherent broadening dynamics become dominant much faster for the simulation with excess noise, the coherence length $L_{c}$ approximately halves from $1 \mathrm{~m}$ for the shot noise limited situation to about $50 \mathrm{~cm}$ for the excess noise simulation.

In contrast, the nonlinear dynamics of the coherent regime are robust against technical noise such as a small amplitude, pulse duration, or central frequency jitter of the pump laser. Such noise simply transfers linearly to small shot-to-shot fluctuations of the SC bandwidth via Eq. (5), but would not experience additional nonlinear noise amplification as it is the case for anomalous dispersion pumping [46]. Indeed, experimental relative intensity noise (RIN) measurements have demonstrated that the noise characteristics of ANDi SC generated in the coherent regime are essentially identical to the RIN of the pump laser [24, 32].

\section{EXPERIMENTAL VERIFICATION}

For the verification of the theoretical predictions presented in the previous sections, we designed an experiment which allows the single-shot detection of SC spectra generated in a commercially available ANDi PCF pumped by picosecond pulses. Similar to the approach of the simulations, the recording of a large number of single-shot spectra enables the analysis of spectrallyresolved fluctuations, which we use to draw conclusions about 
the coherence properties of the generated SC and the involved spectral broadening dynamics.

\section{A. Setup}

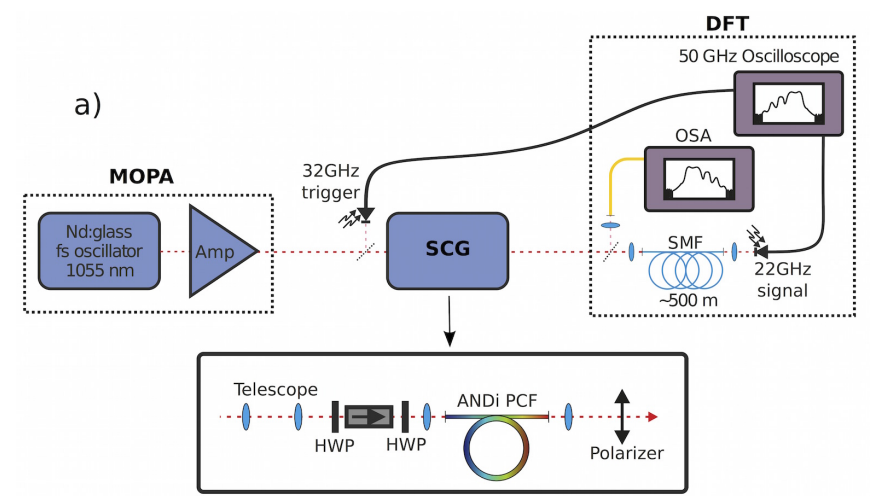

b)
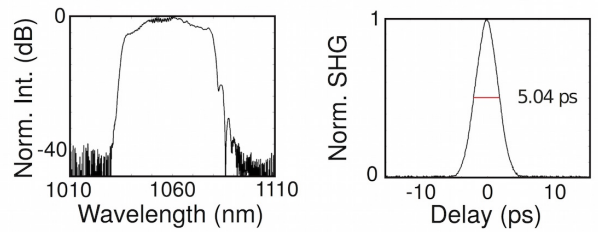

Fig. 10. (a) Schematic experimental setup for single-shot spectral measurements using the dispersive Fourier transform (DFT) method. (b) Spectral and autocorrelation data of the pump pulses after the amplifier. The autocorrelation width of $\sim 5$ ps corresponds to a parabolic FWHM pulse duration of 3.8 ps.

The experimental setup is shown in Fig. 10. We produce the pump pulses using a master oscillator power amplifier (MOPA) system, which employs a Nd:glass modelocked laser (GLX-200, Time Bandwidth) producing $400 \mathrm{fs}, 1.5 \mathrm{~nJ}$ seed pulses at a central wavelength of $1055 \mathrm{~nm}$. An in-house built $\mathrm{Yb}$-doped fiber amplifier operating in the parabolic regime [47] amplifies the seed pulses up to a maximum energy of $50 \mathrm{~nJ}$. The emitted pulses have a spectral bandwidth of $45 \mathrm{~nm}$ at the $-10 \mathrm{~dB}$ level and are chirped to a duration of 3.8 ps FWHM as inferred from autocorrelation data, shown in Fig. 10. The amplifier output is linearly polarized to $13 \mathrm{~dB}$ extinction. The polarization is aligned to the principal axis of the ANDi PCF (NKT NL1050-NEG), whose dispersion profile is very similar to the one considered in the simulations (see Fig. 2). A free-space polarizing isolator is placed before the PCF to prevent back reflections into the amplifier and also functions as a variable attenuator for controlling the coupled pump peak power. At the output of the PCF, a polarizer removes any light which has become depolarized in the PCF. This avoids spectral modulation on the recorded data, which would otherwise result from polarization mode beating at the detector.

The dispersive Fourier transform (DFT) technique [48-50] is used for fast and continuous recording of single shot spectral measurements. This process involves using a highly dispersive optical element after the SCG process to separate the frequency components in time so that they may be resolved by a fast photodiode and oscilloscope. The dispersive element is formed of a $500 \mathrm{~m}$ length of SMF (Corning HI1060) and a $22 \mathrm{GHz}$ photodiode (Agilent A3440C) is used with a $55 \mathrm{GHz}$ oscilloscope (Agilent
Infiniium DCA86100A) to detect the dispersed pulses. A $32 \mathrm{GHz}$ photodiode (Agilent A3440D) is used to trigger the oscilloscope with pulses picked off the main beam at the entrance to the PCF. The time-to-wavelength mapping is performed by calculating the group delay of the HI1060 as a function of wavelength and interpolating the photodiode signal onto a wavelength axis. The measured timing jitter is $<3.75$ ps RMS, corresponding to a wavelength mapping uncertainty of $0.11 \mathrm{~nm}$. The ZDW of the HI1060 fiber is approximately $1300 \mathrm{~nm}$, which imposes a technical limit to the maximum SC bandwidth we can investigate. If the SC extends beyond the ZDW into the anomalous dispersion region of the HI1060 fiber, this causes a degeneracy in the wavelength mapping. Practically we are therefore limited to investigate relatively low peak powers of $P_{0} \leq 2.5 \mathrm{~kW}$, and therefore we used two different fiber lengths to display the range of nonlinear dynamics.

As the photodiode records optical intensity rather than the electric field, the data values obtained with the DFT method are real and the analysis using the complex first order degree of coherence $g_{12}$ of Eq. (1) is inappropriate. The DFT resolves intensity noise, while $g_{12}$ is a measure of phase noise. Nevertheless, there is clearly a strong correlation between intensity and phase noise in normal dispersion SC generation, as evident from the numerical simulations presented in the previous sections, such that intensity fluctuations are good indicators for degrading coherence. Technical noise sources are excluded from the experiments by reducing thermal drift of the free-space to fiber launches at the amplifier output, the PCF launch, and the input to the SMF by mounting the fibers in large aluminium $\mathrm{V}$-grooves which acted as heat sinks. The free-space propagation distances were also kept to a minimum. Additionally, the oscilloscope was optically triggered using a fibre-coupled photodiode for improved stability. The MOPA output is stable within the detection limit of the DFT and the oscilloscope is computer interfaced for fast data acquisition, minimizing the effect of possible long-term drift in the MOPA output. The fluctuations in the DFT signal are therefore attributed to the nonlinear propagation dynamics.

We note that there are important differences between the conditions of the experiments presented in this section and the simulations, and hence it is not possible to make an exact direct comparison. For example, chirped pulses are used from an amplified (MOPA) system. Both a strong, positive and linear spectral phase and a considerable level of excess noise were therefore expected to be present in the experiment. We verified with simulations that the fundamental descriptions presented in the theoretical part of this paper still apply to highly chirped pulses, as will be illustrated below. We also found that assuming a noise level of the MOPA system of $10 \mathrm{~dB}$ above shot noise limit in the simulations reproduces the experimental results very well. Additionally, any small amplitude fluctuations which may have been present in the output from the master oscillator could have been manifest as phase fluctuations due to the high Bintegral of the amplifier, preventing an unavoidable source of technical noise. In spite of these differences, the experiments do reveal tendencies that confirm some aspects of the numerical simulations, in particular the benefit of using $L_{\mathrm{WB}}$ and $L_{R}^{*}$ to estimate the coherence properties of the generated SC.

\section{B. Results and discussion}

The results for two different lengths of ANDi PCF, $1.4 \mathrm{~m}$ and $15.4 \mathrm{~m}$, are summarized in Fig. 11 for varying peak powers coupled into the ANDi PCF. For each peak power, the mean spectrum (black) overlaid with 500 single-shot spectra (grey) are 

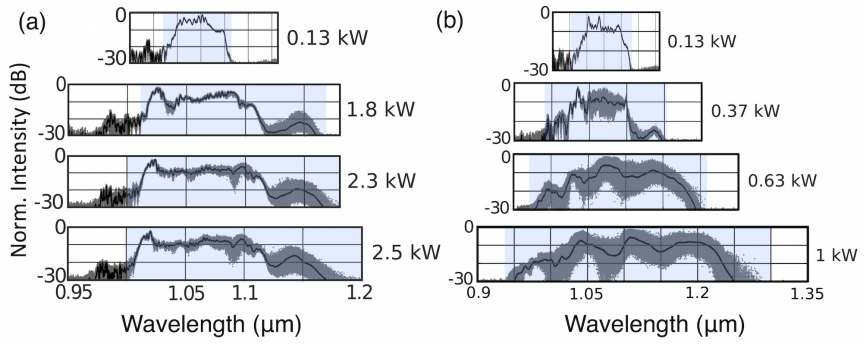

Fig. 11. DFT spectra for the supercontinuum generation in (a) $1.4 \mathrm{~m}$ and (b) $15.4 \mathrm{~m}$ length of ANDi PCF using pump pulses of 3.8 ps duration and varying peak power.
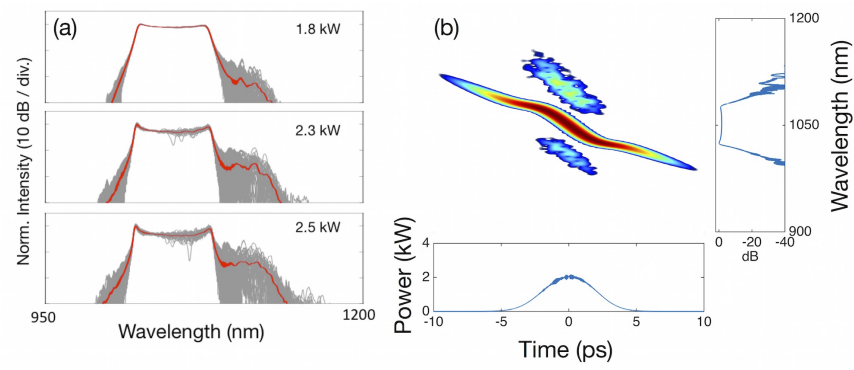

Fig. 12. Numerical simulations for the experimental conditions of Fig. 11 (a), using 1.4 m ANDi PCF and highly chirped input pulses of 3.8 ps duration. We assume excess noise of $10 \mathrm{~dB}$ above shot noise level to account for noise amplification in the MOPA pump system. (a) Simulated mean spectra (red) and fluctuations (grey) at the fiber output for varying pump peak power. (b) Single shot spectrogram for $2.5 \mathrm{~kW}$ pump peak power after $1 \mathrm{~m}$ propagation.

shown to visualize spectral fluctuations. The shading marks the region where the optical signal was high enough for the photodiode to detect it above the noise floor. All fluctuations beyond these regions represent the noise floor of the measurement. We also present numerical simulations in Fig. 12 adapted to the experimental conditions of the $1.4 \mathrm{~m}$ fiber sample, which well reproduce the spectral features and intensity fluctuations observed in the experiments. Differences are a result of increased spectral fine structure of the amplifier output compared to the idealized simulated pulses and uncertainties of the fiber dispersion profile. A comparison of the spectrograms in Figs. 12 (b) and 4 (b) reveals that the development of Stokes and anti-Stokes peaks is not significantly influenced by the strong linear chirp of the pump pulses. However, under experimental conditions the anti-Stokes peak is masked by overlapping components of the main pulse and cannot be directly observed in a spectral measurement. Note that the absence of higher order Stokes and anti-Stokes peaks in Fig. 12 (b) is due to the lower peak power of the pump pulses compared to the conditions in Fig. 4 (b).

In the experiments with the $1.4 \mathrm{~m}$ long fiber sample we investigated pump peak powers in the range $P_{0}=0.13-2.5 \mathrm{~kW}$. The corresponding characteristic length scales are $L_{\mathrm{WB}}=23.5-$ $5.3 \mathrm{~m}$ and $L_{R}^{*}=0.9-0.09 \mathrm{~m}$. Consequently we encounter the situation $L_{R}^{*}<L<L_{\mathrm{WB}}$, indicating a clear dominance of SRS over $\mathrm{SPM} / \mathrm{WB}$. The phase mismatch parameter $K=(-8.5)-(-0.5)$ shows only weak to moderate coupling of SRS and FWM. We therefore expect the development of clear SRS signatures and decoherence mechanisms similar to the dynamics discussed in
Sec. 4.B, i.e. gradual coherence collapse from long to short wavelengths. Fig. 11 (a) confirms these expectations. While the main spectral band around the pump wavelength broadens only modestly due to SPM and initially remains stable, a distinct Raman Stokes peak exhibiting large shot-to-shot fluctuations develops at $1150 \mathrm{~nm}$ for $P_{0}=1.8 \mathrm{~kW}$. Taking into account the peak Raman gain shift of $\Omega_{R}=2 \pi \times 13.2 \mathrm{THz}$, the pump for the observed Stokes peak is located at approximately $1095 \mathrm{~nm}$, i.e. near the most red-shifted SPM peaks. This supports the numerical finding in Sec. 4.B that SRS first develops at the most red-shifted SPM peak where the spectro-temporal intensity is highest. We also observe a broadening of the SRS peak with increasing $P_{0}$, which we attribute to the decreasing $L_{R}^{*}$ such that the SRS onset occurs at earlier propagation distances where SPM broadening is reduced. The broadening of the Stokes peak is then a direct consequence of the increased spectro-temporal reshaping of the pulse due to SPM and the associated spectral shift of the maximum Raman gain during propagation, as discussed in Sec. 5. Fluctuations in the main spectral band begin at the most red-shifted SPM components and spread towards shorter wavelengths with increasing pump power. These interpretations are also confirmed by the numerical simulations under experimental conditions.

Since $L \ll L_{W B}$ for this short fiber sample, we could not observe the influence of WB on the broadening dynamics. We therefore repeated the experiments with an increased fiber length of $15.4 \mathrm{~m}$. As expected, the results displayed in Fig. 11 (b) reveal that the longer propagation distance leads to a decrease of the SRS power threshold to $P_{0}=370 \mathrm{~W}$, where also the main spectral band around the pump wavelength already exhibits strong fluctuations. At $P_{0}=630 \mathrm{~W}$, we can identify the signature of an anti-Stokes Raman pulse at a central wavelength of approximately $1000 \mathrm{~nm}$, as predicted in the simulations in Sec. 3, confirming the coupling of SRS and FWM as well as its role in the noise distribution process towards wavelengths shorter than the pump. The anti-Stokes peak is observable now due to the reduced SPM broadening compared to the experiments with the shorter fiber sample. At the $P_{0}=630 \mathrm{~W}$ level, $L_{\mathrm{WB}}$ has dropped to $10.5 \mathrm{~m}$ and consequently we observe the characteristic flattening of the long wavelength edge indicating the onset of WB. The new wavelengths in the spectral wing are generated incoherently, confirming the numerical observation of incoherent optical wave breaking discussed in Sec. 4.B caused by the involvement of the SRS pulse in the WB process. With a further increase in $P_{0}$ incoherent $\mathrm{WB}$ is also observed on the short wavelength edge and the entire spectrum exhibits large intensity fluctuations. This agrees well with the formation of an incoherent "cloud" observed in the spectrogram representations in Sec. 4.

\section{CONCLUSIONS}

We reported and explained the rich variety of nonlinear dynamics occurring in the largely unexplored transition region between coherent and incoherent nonlinear spectral broadening during supercontinuum formation in the (all-) normal dispersion region of optical fibers. For pumping with high peak power pulses leading to octave-spanning SC spectra we showed that high temporal coherence can be maintained up to pump pulse durations of up to $1.5 \mathrm{ps}$, corresponding to initial soliton numbers of $N \simeq 600$, while longer pulses generally lead to incoherent spectral broadening. In terms of $N$, this corresponds to an approximately 50 times increase of the coherent regime compared to anomalous dispersion pumping. Hence, for the first time, 
we propose a realistic scheme for coherent SC generation with pump pulse durations in the picosecond regime.

We could clarify the double role played by the coupling of parametric FWM and SRS: in the incoherent regime it is responsible for the non-phase-matched parametric amplification of noise via generation of strong anti-Stokes Raman components, which we identified as the dominating effect for noise distribution towards wavelengths shorter than the pump. In contrast, in the coherent regime the FWM/SRS coupling leads to gain saturation for noise amplification at high pump powers, which contributes significantly to the excellent coherence properties of SC pulses generated in ANDi fibers at short pulse durations.

We further explored the effects arising from the interaction of coherent and incoherent dynamics, and showed that spectral decoherence can occur in two different ways: (i) instantly, i.e. spectral components are directly generated incoherently, or (ii) gradually, i.e. the coherence of spectral components collapses slowly with propagation distance. The former is observed, for example, during the generation of distinct Raman Stokes and anti-Stokes pulses by direct noise amplification, or during incoherent optical wave breaking due to the parametric interaction of coherent and incoherent components. The latter we observed during incoherent cloud formation, where the coherence of a fully developed SC pulse gradually collapses with propagation distance. While at low pump peak powers we could distinguish coherent and instantly generated incoherent components by their distinct spectral signatures, both decoherence mechanisms are entirely concealed at high powers and cannot be identified by spectral measurements. Care should hence be taken to avoid the frequent argumentation that SC generated in normal dispersion fibers exhibiting the characteristic spectral shape of coherent SC (flat top and steep edges) are necessarily coherent. More careful experimental measurements, such as the DFT technique applied in this paper, are required to make an reliable assessment of the SC coherence properties.

Finally, our results indicate that SC coherence properties can be influenced by fiber design, since the coupling strength of FWM and SRS depends on the dispersive characteristics of the fiber. The low and flat normal dispersion design employed for ANDi fibers to maximize the SC spectral bandwidth is also conducive for maintaining good temporal coherence properties in the short pulse regime. This finding might also prove relevant for the design of optical fibers for short-pulse fiber amplifiers, where it is usually desirable to suppress incoherent nonlinear dynamics, such as the build-up of significant Raman-amplified noise.

Funding. Engineering and Physical Sciences Research Council (EPSRC). Royal Academy of Engineering. Swiss National Science Foundation (SNSF) (NCCR MUST).

\section{REFERENCES}

1. R. Alfano, ed., The supercontinuum laser source (3rd edition) (Springer, New York, 2016).

2. J. M. Dudley and J. R. Taylor, eds., Supercontinuum generation in optical fibers (Cambridge University Press, 2010).

3. R. Holzwarth, T. Udem, T. W. Hänsch, J. C. Knight, W. J. Wadsworth, and P. S. J. Russell, "Optical frequency synthesizer for precision spectroscopy," Phys. Rev. Lett. 85, 2264-2267 (2000).

4. T. Udem, R. Holzwarth, and T. W. Hänsch, "Optical frequency metrology," Nature 416, 233-237 (2002).

5. N. Nishizawa, Y. Chen, P. Hsiung, E. P. Ippen, and J. G. Fujimoto, "Real-time, ultrahigh-resolution, optical coherence tomography withan all-fiber, femtosecond fiber laser continuum at $1.5 \mu \mathrm{m}$," Opt. Lett. 29, 2846-2848 (2004)

6. G. Humbert, W. Wadsworth, S. Leon-Saval, J. Knight, T. Birks, P. S. J. Russell, M. Lederer, D. Kopf, K. Wiesauer, E. Breuer, and D. Stifter, "Supercontinuum generation system for optical coherence tomography based on tapered photonic crystal fibre," Opt. Express 14, 1596-1603 (2006).

7. H. Tu and S. A. Boppart, "Coherent anti-Stokes Raman scattering microscopy: overcoming technical barriers for clinical translation," J. Biophoton. 7, 9-22 (2014).

8. A. M. Heidt, A. Hartung, and H. Bartelt, The Supercontinuum Laser Source: The Ultimate White Light (Springer New York, New York, NY, 2016), chap. Generation of Ultrashort and Coherent Supercontinuum Light Pulses in All-Normal Dispersion Fibers, pp. 247-280.

9. H. Tu and S. A. Boppart, "Coherent fiber supercontinuum for biophotonics," Laser \& Photon. Rev. 7, 628-645 (2013).

10. A. M. Heidt, A. Hartung, G. W. Bosman, P. Krok, E. G. Rohwer, $\mathrm{H}$. Schwoerer, and H. Bartelt, "Coherent octave spanning near-infrared and visible supercontinuum generation in all-normal dispersion photonic crystal fibers," Opt. Express 19, 3775-3787 (2011).

11. Y. Liu, H. Tu, W. A. Benalcazar, E. J. Chaney, and S. A. Boppart, "Multimodal nonlinear microscopy by shaping a fiber supercontinuum from 900 to 1160 nm," IEEE J. Sel. Top. Quantum Electron. 18, 12091214 (2012).

12. Y. Liu, M. D. King, H. Tu, Y. Zhao, and S. A. Boppart, "Broadband nonlinear vibrational spectroscopy by shaping a coherent fiber supercontinuum," Opt. Express 21, 8269-8275 (2013).

13. A. M. Heidt, J. Rothhardt, A. Hartung, H. Bartelt, E. G. Rohwer, J. Limpert, and A. Tünnermann, "High quality sub-two cycle pulses from compression of supercontinuum generated in all-normal dispersion photonic crystal fiber," Opt. Express 19, 13873-13879 (2011).

14. S. Demmler, J. Rothhardt, A. M. Heidt, A. Hartung, E. G. Rohwer, H. Bartelt, J. Limpert, and A. Tünnermann, "Generation of high quality, 1.3 cycle pulses by active phase control of an octave spanning supercontinuum," Opt. Express 19, 20151-20158 (2011).

15. B. Schenkel, R. Paschotta, and U. Keller, "Pulse compression with supercontinuum generation in microstructure fibers," J. Opt. Soc. Am. B 22, 687-693 (2005).

16. M. Nakazawa, K. Tamura, H. Kubota, and E. Yoshida, "Coherence degradation in the process of supercontinuum generation in an optical fiber," Opt. Fiber Technol. 4, 215 - 223 (1998).

17. J. M. Dudley, G. Genty, and S. Coen, "Supercontinuum generation in photonic crystal fiber," Rev. Mod. Phys. 78, 1135-1184 (2006).

18. J. M. Dudley and S. Coen, "Coherence properties of supercontinuum spectra generated in photonic crystal and tapered optical fibers," Opt. Lett. 27, 1180-1182 (2002).

19. X. Gu, L. Xu, M. Kimmel, E. Zeek, P. O'Shea, A. P. Shreenath, R. Trebino, and R. S. Windeler, "Frequency-resolved optical gating and singleshot spectral measurements reveal fine structure in microstructure-fiber continuum," Opt. Lett. 27, 1174-1176 (2002).

20. K. L. Corwin, N. R. Newbury, J. M. Dudley, S. Coen, S. A. Diddams, K. Weber, and R. S. Windeler, "Fundamental noise limitations to supercontinuum generation in microstructure fiber," Phys. Rev. Lett. 90, 113904 (2003).

21. X. Gu, M. Kimmel, A. Shreenath, R. Trebino, J. Dudley, S. Coen, and R. Windeler, "Experimental studies of the coherence of microstructurefiber supercontinuum," Opt. Express 11, 2697-2703 (2003).

22. G. Genty, S. Coen, and J. M. Dudley, "Fiber supercontinuum sources (invited)," J. Opt. Soc. Am. B 24, 1771-1785 (2007).

23. A. M. Heidt, "Pulse preserving flat-top supercontinuum generation in all-normal dispersion photonic crystal fibers," J. Opt. Soc. Am. B 27, 550-559 (2010).

24. N. Nishizawa and J. Takayanagi, "Octave spanning high-quality supercontinuum generation in all-fiber system," J. Opt. Soc. Am. B 24, 1786-1792 (2007).

25. L. E. Hooper, P. J. Mosley, A. C. Muir, W. J. Wadsworth, and J. C. Knight, "Coherent supercontinuum generation in photonic crystal fiber with all-normal group velocity dispersion," Opt. Express 19, 4902-4907 
(2011)

26. Y. Liu, H. Tu, and S. A. Boppart, "Wave-breaking-extended fiber supercontinuum generation for high compression ratio transform-limited pulse compression," Opt. Lett. 37, 2172-2174 (2012).

27. S. Coen, A. H. L. Chau, R. Leonhardt, J. D. Harvey, J. C. Knight, W. J. Wadsworth, and P. S. J. Russell, "Supercontinuum generation by stimulated raman scattering and parametric four-wave mixing in photonic crystal fibers," JOSA B 19, 753-764 (2002).

28. H. Ma, X. Zhang, Q. Jing, Y. Huang, and X. Ren, "Effect of intrapulse raman scattering on broadband amplitude noise of supercontinuum generated in fiber normal dispersion region," Applied optics 51, 19621967 (2012).

29. M. Klimczak, G. Soboń, K. Abramski, and R. Buczyński, "Spectral coherence in all-normal dispersion supercontinuum in presence of raman scattering and direct seeding from sub-picosecond pump." Optics Express 22, 31635-31645 (2014).

30. H. Tu, Y. Liu, X. Liu, D. Turchinovich, J. Lægsgaard, and S. A. Boppart, "Nonlinear polarization dynamics in a weakly birefringent all-normal dispersion photonic crystal fiber: toward a practical coherent fiber supercontinuum laser," Opt. Express 20, 1113-1128 (2012).

31. S. R. Domingue and R. A. Bartels, "Overcoming temporal polarization instabilities from the latent birefringence in all-normal dispersion, wavebreaking-extended nonlinear fiber supercontinuum generation," Opt. Express 21, 13305-13321 (2013).

32. Y. Liu, Y. Zhao, J. Lyngsø, S. You, W. L. Wilson, H. Tu, and S. A. Boppart, "Suppressing short-term polarization noise and related spectral decoherence in all-normal dispersion fiber supercontinuum generation," Journal of Lightwave Technology 33, 1814-1820 (2015).

33. A. M. Heidt, "Efficient adaptive step size method for the simulation of supercontinuum generation in optical fibers," J. Lightwave Tech. 27, 3984-3991 (2009).

34. A. A. Rieznik, A. M. Heidt, P. G. Konig, V. A. Bettachini, and D. F. Grosz, "Optimum integration procedures for supercontinuum simulation," IEEE Photonics Journal 4, $552-560$ (2012).

35. http://www.freeopticsproject.org.

36. M. Koshiba and K. Saitoh, "Applicability of classical optical fiber theories to holey fibers," Opt. Lett. 29, 1739-1741 (2004).

37. K. Saitoh and M. Koshiba, "Empirical relations for simple design of photonic crystal fibers," Opt. Express 13, 267-274 (2005).

38. G. Agrawal, Nonlinear Fiber Optics (Academic Press, 2007).

39. C. Finot, B. Kibler, L. Provost, and S. Wabnitz, "Beneficial impact of wave-breaking for coherent continuum formation in normally dispersive nonlinear fibers," J. Opt. Soc. Am. B 25, 1938-1948 (2008).

40. N. Bloembergen and Y. Shen, "Coupling between vibrations and light waves in raman laser media," Physical Review Letters 12, 504 (1964).

41. Y. R. Shen and N. Bloembergen, "Theory of stimulated brillouin and raman scattering," Physical Review 137, A1787 (1965).

42. S. Coen, D. A. Wardle, and J. D. Harvey, "Observation of non-phasematched parametric amplification in resonant nonlinear optics," Phys. Rev. Lett. 89, 273901 (2002).

43. S. Trillo and S. Wabnitz, "Parametric and raman amplification in birefringent fibers," J. Opt. Soc. Am. B 9, 1061-1082 (1992).

44. E. A. Golovchenko, P. V. Mamyshev, A. N. Pilipetskii, and E. M. Dianov, "Mutual influence of the parametric effects and stimulated raman scattering in optical fibers," IEEE Journal of Quantum Electronics 26, 1815-1820 (1990).

45. N. R. Newbury and W. C. Swann, "Low-noise fiber-laser frequency combs (invited)," J. Opt. Soc. Am. B 24, 1756-1770 (2007).

46. N. Newbury, B. Washburn, K. Corwin, and R. Windeler, "Noise amplification during supercontinuum generation in microstructure fiber," Optics Letters 28, 944-946 (2003).

47. M. Fermann, V. Kruglov, B. Thomsen, J. Dudley, and J. Harvey, "Selfsimilar propagation and amplification of parabolic pulses in optical fibers," Physical Review Letters 84, 6010 (2000).

48. T. Jannson, "Real-time fourier transformation in dispersive optical fibers," Optics letters 8, 232-234 (1983).

49. B. Wetzel, A. Stefani, L. Larger, P.-A. Lacourt, J.-M. Merolla, T. Sylvestre, A. Kudlinski, A. Mussot, G. Genty, F. Dias et al., "Real- time full bandwidth measurement of spectral noise in supercontinuum generation," Scientific reports 2 (2012).

50. K. Goda and B. Jalali, "Dispersive fourier transformation for fast continuous single-shot measurements," Nature Photonics 7, 102-112 (2013). 